\title{
Plasmonic colour laser printing
}

Zhu, Xiaolong; Vannahme, Christoph; Højlund-Nielsen, Emil; Mortensen, N. Asger; Kristensen, Anders

Published in:

Nature Nanotechnology

Link to article, DOI:

10.1038/nnano.2015.285

Publication date:

2016

Document Version

Peer reviewed version

Link back to DTU Orbit

Citation (APA):

Zhu, X., Vannahme, C., Højlund-Nielsen, E., Mortensen, N. A., \& Kristensen, A. (2016). Plasmonic colour laser printing. Nature Nanotechnology, 11(4), 325-329. https://doi.org/10.1038/nnano.2015.285

\section{General rights}

Copyright and moral rights for the publications made accessible in the public portal are retained by the authors and/or other copyright owners and it is a condition of accessing publications that users recognise and abide by the legal requirements associated with these rights.

- Users may download and print one copy of any publication from the public portal for the purpose of private study or research.

- You may not further distribute the material or use it for any profit-making activity or commercial gain

- You may freely distribute the URL identifying the publication in the public portal

If you believe that this document breaches copyright please contact us providing details, and we will remove access to the work immediately and investigate your claim. 


\section{Plasmonic colour laser printing}

Xiaolong Zhu ${ }^{1 *}$, Christoph Vannahme ${ }^{1}$, Emil Højlund-Nielsen ${ }^{1}$, N. Asger Mortensen ${ }^{2 *} \&$ Anders Kristensen ${ }^{1 *}$

${ }^{1}$ Department of Micro and Nanotechnology, Technical University of Denmark, DK-2800 Kongens Lyngby, Denmark

${ }^{2}$ Department of Photonics Engineering, Technical University of Denmark, DK-2800 Kongens Lyngby, Denmark

Colour generation by plasmonic nanostructrues ${ }^{1,2}$ and metasurfaces ${ }^{3,4}$ has several advantages over dye technology: reduced pixel area, sub-wavelength resolution and the production of bright and non-fading colours ${ }^{5}$. However, plasmonic colour patterns need to be predesigned and printed either by e-beam lithography (EBL $)^{6-11}$ or focused ion beam (FIB $)^{12-14}$, both expensive and not scalable processes that are not suitable to post-processing customization. Here we show a method of colour printing on nanoimprinted plasmonic metasurfaces using laser post-writing. Laser pulses induce transient local heat generation that leads to melting and reshaping of the nanostructures ${ }^{15}$. Depending on the laser pulse energy density, different surface morphologies that support different plasmonic resonances leading to different colour appearances can be created. Using this technique we can print all primary colours with a speed of $1 \mathrm{~ns}$ per pixel, resolution up to 127,000 dots per inch (DPI) and power consumption down to $0.3 \mathrm{~nJ}$ per pixel.

The concept of our laser printing is illustrated in Fig. 1. The printable plasmonic metasurface 
is composed of metal disks on top of dielectric pillars, hovering above a holey metal film, see Fig. 1a and $1 \mathrm{~b}$. This design with polymeric pillars can be implemented by nano-imprinting, roll-to-roll processing or injection molding technologies based on a master defined by EBL. We use abundant and recyclable aluminum (Al) as the plasmonic material, which exhibits high plasma frequency and has recently been studied to yield surface plasmon resonances (SPRs) from visible to UV ${ }^{16,17}$. Abundant polymers and hybrid materials suitable for nanoimprinting can be used. For instance, PMMA (Polymethylmethacrylat) or Ormocomp (Micro resist technology GmbH) are good candidates, which can be imprinted in thermal nanoimprint (PMMA) or UV curing (Ormocomp) processes. Due to the plasmon hybridization of the disk-hole system in the Al metasurface ${ }^{18}$, reflected colours caused by resonant absorptions at specific wavelengths can be modified by changing the geometric parameters ${ }^{6,19}$ (see Supplementary Figure S1).

For a laser pulse of nanoseconds duration, the instant energy is efficiently high to heat the selected area above the melting temperature. The disks may thus transform their shapes into thicker disks or spheres in a crystallographic phase transition process (Fig. 1c) ${ }^{20,21}$, and finally they may be ablated away. By the excitation of SPR with electric field confinement, light energy is redistributed and concentrated in specific regions of the disk-hole unit cell, enabling a fine tuning of the morphology by adjusting the input pulse energy density. In return, the change of morphology shifts the resonant frequency of the SPR, leading to a variation in reflected colours. This allows printing of colours on a specific plasmonic surface by controlling the laser parameters (such as power, spot size, frequency etc.) as well as the position of the laser spot (see Supplementary Video I). 
To realize a desired colour from laser irradiation, we modify the SPR through single-pulse laser exposure ( $1 \mathrm{~ns}, \lambda=532 \mathrm{~nm}$ ). As the input laser energy dosage is increased from 0 to $535 \mathrm{~nJ}$ in the focal plane of a $4 \times$ objective (spot size $\sim 50 \mu \mathrm{m}$ in diameter), the main SPR shifts from $600 \mathrm{~nm}$ to $500 \mathrm{~nm}$ and the printed colour varies from cyan to yellow (Fig. 2a). We further explore the continuous tuning of colours across the visible spectrum in metasurfaces with different parameters (Supplementary Figures S2 and S3). Remarkably, CMY primaries (cyan, magenta and yellow) used in traditional colour printing are obtained comprehensively. With sufficient laser intensity, the $\mathrm{Al}$ nanodisks can be annealed at their rims and evolved into spherical objects (Fig. $2 \mathrm{~b}$ and Supplementary Figure S4). Also, by increasing the laser intensity, the spheres are ablated from the polymer interface, owing to a rapid change in the center of mass during the melting process which provides the particles with a repulsive momentum ${ }^{22}$. Furthermore, for a laser energy above $500 \mathrm{~nJ}$, the holes in the continuous film on the substrate can even be enlarged in a controlled manner.

To describe the morphology transformation of the Al disks, we offer a simplified model of the complex thermodynamic phase transition. Neglecting gravity, we vary the thickness $t$ of roundcornered disks (or the radius $r$ of the sphere) while preserving the over-all metal volume of the initial disks. Simulations with this model (Fig. 2c) predict the blue-shift of SPR and demonstrate a qualitative agreement with the corresponding measurements for different thermo-transition states. It should be noted that the measured spectra show a broadening of the resonances when comparing with the simulated ones because of the defects in printing (Supplementary Figure S5). From the simulated electric field enhancements for printed metasurfaces (Fig. 2d), we find that the electric field decreases during the gradual transformation from disks to spheres. The field is strongly 
localized at the sharp corners of the unchanged disks (Fig. 2d (i)), dissipating in the intermediate shape (Fig. 2d (ii)) and finally dispersively covering the sphere (Fig. 2d (iii)). The absorbed heat power is directly related to the electric field distributed in the materials and can simply be expressed as

$$
Q_{a b s}(\omega)=\frac{1}{2} \omega \int \varepsilon^{\prime \prime}(\omega)|\mathbf{E}(\omega)|^{2} d V
$$

where $\omega$ is the angular frequency, $\varepsilon^{\prime \prime}(\omega)$ is the imaginary part of the dielectric function $\varepsilon=\varepsilon^{\prime}+i \varepsilon^{\prime \prime}$, $\mathbf{E}$ is the electric field, and the integration is carried out over the volume of the unit cell. Thus, the heat absorption can obviously be improved by overlapping the lossy metal with a strong electric field. With the aid of the SPR, the electrical field is strongly localized and enhanced near the metal surface and exponentially decays into the surrounding medium, causing a strong heat power confined at the interface ${ }^{23}$. This process can be further understood by examining the simulated temperature evolutions during the first initial nanoseconds for different structural morphologies (Fig. 2e) and the temperature distribution resulting from thermo-plasmonic heating (Fig. 2f), see also Supplementary Figure S6.

Based on the trait of local-plasmon heating, the plasmonic metasurfaces can be embedded in transparent polymers for laser printing (Fig. 3a) ${ }^{24}$. With the excitation of the SPR, the plasmon enhanced photo-thermal melting ensures that the writing process only takes place at the plasmonic metasurface within the focal plane. As shown in Fig. 3a, the coating leads to a redshift of the resonances in the system and a corresponding colour change before and after laser printing because of the increased refractive index of the surroundings. A top coating offers the advantage to protect the structure from mechanical damage, fingerprints, greasy residue, and so forth, making the 
proposed "plasmonic paper" more robust and fully flexible for colour printing in everyday applications (Fig. 3b) . Impressively, by embedding $20 \mathrm{~nm} \mathrm{Al}$ inside plastics, our printing method creates environmentally sound colouration solutions for consumer products (such as beverage bottle, toy and automotive), with only a ppm (parts-per-million) level residue of $\mathrm{Al}$ for recycling.

For a detailed expression of a colour image, it is necessary not only to produce single colour depth, but also colour mixing ${ }^{9,25}$ or overlapping (Supplementary Figure S7), to broaden the capability of our colour library. For a proof-of-concept demonstration, spatial colour mixing is carried out by coordinating the distribution of colour dots. By controlling the laser spot size and step dimension, we printed magenta colour dots onto the cyan background with different filling factors. The spot size is well below the resolution of a regular human eye so that the spatially distributed dots can be seen as a single mixed blue-violet colour (Fig. 3c and 3d). A simple averaging of two different colour spectra yields a similar result as the reflection spectrum of the mixed colour, proving the spatial mixing of distinct structural colours (Fig. 3e and Supplementary Figure S8). Note that the spectrum of the mixed colour is slightly different from the theoretical prediction of $50 \%$ mixture because of the fluctuation of the spot size. Despite the practical advantages of laser manipulating, colour generation by spatial mixing as a complementary strategy is ultimately necessary to accomplish full-colour printing ${ }^{26}$. In principle, a full-colour library is possible by combining the already-rich colours (caused by laser reshaping directly) and the additional colour mixing possibilities. Besides, monochrome laser printing on the colourless plasmonic paper is also possible (Supplementary Figure S9). 
We further expound how to increase the printing resolution to exceed the diffraction limit. Conventional optics suffer from the diffraction limit, meaning that a spatial resolution below a quarter of a micrometer seems entirely out of reach for visual applications. Interestingly, photon heating occurs only in the vicinity of the focal spot and the size of melted material can be reduced due to the Gaussian probability distribution of the photon fluence density. This property leads to a critical value of exposure energy where the melting reaction is initiated, which essentially defines a threshold that excludes the low-intensity wings from melting and thus reduces the size ${ }^{27}$, as displayed in the upper panel in Fig. 4a. However, a small (e.g. 10\%) fluctuation of the laser intensity would be detrimental for this mechanism. Surface plasmons possess two natural properties of subwavelength light confinement and intense field enhancement ${ }^{28}$. Applying a plasmonic super-lens, sub-diffraction-limited imaging with 60 nanometer half-pitch resolution has been implemented ${ }^{29}$. Here, we push the laser-induced colour printing to the sub-diffraction scale by exploiting the plasmonic thermal reshaping. As indicated in the lower panel in Fig. 4a, electric field is confined and enhanced at the plasmonic metasurface. The local field enhancements help to localize the light intensity, thereby causing localized heating at the intended particle. This mechanism is the key to successfully realize (1) a higher spatial resolution, as the melting at the unit cell can be well controlled within the disk, (2) a better stability, because only a laser fluctuation $\geq 100 \%$ can heat and melt the nearest neighboring unit cell, and (3) a lower power, where a $1 \%$ of original laser power is feasible due to the 100 times enhancements of the E-field intensity.

We are thus able to design laser printed colour pixels of arbitrary small size by choosing an appropriate but relatively low laser-pulse energy. To strengthen our conclusion, we applied a single 
nanosecond laser pulse focused through a 0.65 NA $40 \times$ objective (spot size $\sim 4 \mu \mathrm{m}$ ) for printing. Considering a Gaussian distribution of the laser energy, only a small central region can reach the melting threshold, as manifested in the circles in Fig. 4b. We have achieved laser printing at the single unit cell level of the metasurface with a periodicity of $200 \mathrm{~nm}$, which is smaller than the theoretical diffraction limit throughout the entire visible spectrum, as shown in the red circle in Fig. 4b. Furthermore, the actual melting part of a disk can be less than $50 \mathrm{~nm}$ when employing a single-pulse energy in a spot down to $0.3 \mathrm{~nJ}$ (the inset in Fig. 4b), resulting in the laser-induced manipulation of structures within the single unit cell. The reshaping part of the disk can even be smaller under a weaker intensity (see the results in Supplementary Figure S10).

As the plasmonic heating flattens the sharp corners from the disk-hole system and eliminates the narrow nanogaps in between, the electromagnetic field enhancement is consequently weakened. The gradient change of geometry and the attenuation of intensity actually protect the printing area from reacting to multiple-pulse irradiation, as the first pulse degrades the geometry immediately, making the field intensities upon subsequent pulses being below the threshold value. Moreover, the intensity gaps between these transition states of morphologies provides distinct power levels for reshaping, which provides easier colour switching, mixing and overlapping, as shown in Fig. $4 c$.

To illustrate the capability to print arbitrary images with colour and tonal control, we printed a selected image using a single nanosecond laser pulse focused through a 0.8 NA $50 \times$ objective lens (spot size $\sim 3 \mu \mathrm{m}$ ). In order to prove the ultimate capability, we actualized a colour design 
in blue tone (see also Supplementary Figure S10 and S11) and a step size of $200 \mathrm{~nm}$ (the same dimension as the unit cell). The printed image was taken by an optical microscope with a 0.9 NA $100 \times$ objective lens. As the pixel is at the theoretical resolution limit of the optical microscope, printed pixels can only be distinguished in a blurred way (Fig. 4d). In Fig. 4e we exhibit laser prints of 9 images in different colours, which were taken by the same microscope with a 0.9 NA 100X objective lens. Although we have taken a controlled laser power for patterning, the printing can also be performed with up to 5 colours with sub-diffraction-limit resolution and the high sharpness (Fig. 4e). In particular, the images presented in Figures 4d and 4e with about 125,000 pixels in total would fit into the cross-section of a human hair. As any traditional printer, our plasmonic colour laser printer can perform printing technologies (such as half-toning) in a super-high resolution (Fig. 4f). Moreover, it is worth noting that the image could also be laser-printed through a mask (Supplementary Figure S12), a spatially modulated laser array or scanning mirrors, which would allow a faster printing with a resolution approaching the single-unit-cell limit by following the achievements in this work. With the multi-colour printing on plastic-based plasmonic metasurfaces, multi-nary code recording can be performed, paving the way for a low-cost recording apparatus (Supplementary Figure S13). By incorporating the sub-wavelength recording and multinary plasmonic colour channels, a disk capacity of TB level for a DVD sized disk with a single metasurface could potentially be realized.

In summary, we have demonstrated full-colour laser printing on plasmonic metasurfaces with sub-diffraction-limit resolution. The nanoimprinted metasurfaces are composed by a $20 \mathrm{~nm}$ Al film buried in a thin-film polymer, which is flexible, economic and recyclable. Reconfigured 
by plasmon resonances, our laser printing process allows for recording speeds up to $1 \mathrm{Gbit} \mathrm{s}^{-1}$ with a spot energy down to $0.3 \mathrm{~nJ}$. Colours are printed when only a single unit cell is modified by laser heating with individual pixels of $200 \mathrm{~nm} \times 200 \mathrm{~nm}$ squares, thus enabling laser printing of colours at a resolution of $\sim 127,000$ DPI. This technology promises exciting avenues from largescale colour printing and decoration to nanoscale colour patterning, encryption and data storage, where high information densities are pursued.

\section{Methods}

Sample preparation. Firstly, a silicon master mold was fabricated by applying electron-beam lithography (EBL, JEOL JBX-9500FS $100 \mathrm{keV}$ prototype) and dry etching. We used a fast single spot writing technique, where EBL with a focused Gaussian beam was used to define shapes directly. The single-spot exposure strategy uses the EBL system as a raster scan tool to write a large area, when a beam step size is larger than the spot size ${ }^{30}$. The sample was fabricated on a $0.5 \mathrm{~mm}$ thick 4 inch Borofloat glass wafer where upon a thin film of Ormocomp mixed with ma-T 1050 thinner $(25 \% \mathrm{w} / \mathrm{w}$, both micro resist technology) was dispensed. The silicon stamp coated with an antistiction coating was employed for replicating the pillar structure via room temperature nanoimprint into the Ormocomp layer. The Ormocomp film was cured by exposure to UV light and separated from the silicon master and peeled from the Borofloat glass substrate afterwards. Then, a $20 \mathrm{~nm} \mathrm{Al} \mathrm{film} \mathrm{was} \mathrm{deposited} \mathrm{by} \mathrm{an} \mathrm{electron} \mathrm{beam} \mathrm{evaporator} \mathrm{(Alcatel} \mathrm{SCM} \mathrm{600)} \mathrm{at} 5 \AA / \mathrm{s}$ after imprint under a process pressure of $10^{-6}-10^{-5}$ mbar. For polymer coated samples, a sufficiently thick layer of PMMA (10\% 950PMMA in Anisole, MicroChem Corp) was spin-coated (500 rpm, 
$\sim 10 \mu \mathrm{m})$ on top to avoid Fabry-Pérot interference.

Optical setups. The optical setup for laser colour printing is schematically illustrated in Supplementary Figure S14a. It comprises a Nikon Ti-U inverted microscope where a laser (CryLaS FDSS532-150) emitting $1 \mathrm{~ns}$ pulses at $532 \mathrm{~nm}$ is used for printing. The laser pulse energy is controlled with a half wave plate on a computer controlled rotation stage combined with a polarizing beam splitter. The laser beam is focused onto the input port of a module of the microscope which is made to accept fiber-coupled laser input sources for diffraction-limited focusing onto the sample. A white light source can be coupled in for illumination as well, not shown in the figure. In this work, the sample was mounted on a computer controlled piezo nanopositioning stage (Mad City Labs Nano H50 series piezo electric stage, $0.1 \mathrm{~nm}$ resolution, $50 \mu \mathrm{m}$ travel, $\mathrm{XY}$ axes), which was placed on a computer controlled motor stage. The optical setup for spectroscopic imaging is illustrated in Supplementary Figure S14b. A high intensity white light source (Energetiq EQ-99XFC fiber coupled laser driven light source) is used for illumination. An image of the surface is projected onto the control CCD array, CCD1. Via a beam splitter, a duplicated image of the device surface is projected onto the entrance slit of an imaging spectrometer with a $150 \mathrm{~g} / \mathrm{mm}$ grating (Acton SP-2756 imaging spectrograph with PIXIS100B digital CCD camera, $100 \times 1340$ pixels, CCD2). Optical micrographs were acquired using a Nikon Eclipse L200 microscope with a Nikon digital camera (DS-Fi1). Photographs were taken by a Canon IXUS camera.

Laser printing. For spot-by-spot printing, a function generator was used to select single $1 \mathrm{~ns}$ 
pulses from the pulse train of the laser with the power tuned accordingly for each spot. Motor/piezo stages, rotators and laser controllers were connected to a computer via I/O equipment. A Matlab code was used to switch the laser on and off and select the repetition rate via the function generator, control the laser energy via the half-wave plate and the polarizing beam splitter, and move the piezo/motor stages for laser printing of images in a raster scan.

Numerical simulation. The plasmonic metasurface was simulated by CST (www.CST.com) microwave studio software. Reflection spectra and field distributions of structures under different conditions (thermal reshaping, oxide coating and so on) were simulated by a finite element method. An EM-thermal co-simulation approach was applied to solve the coupled electromagnetic and heat transfer problem. The results are presented and further discussed in Supplementary Information.

\section{Figures}


a

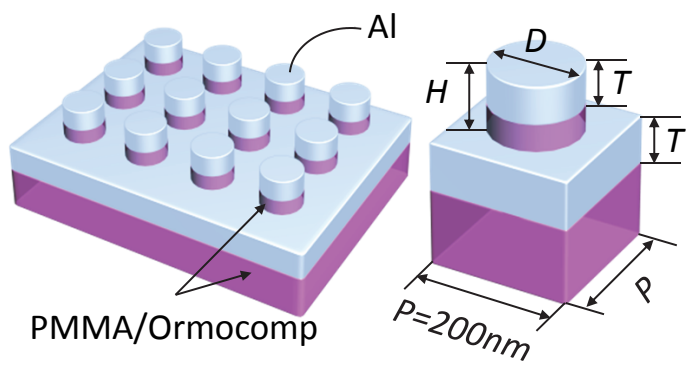

b

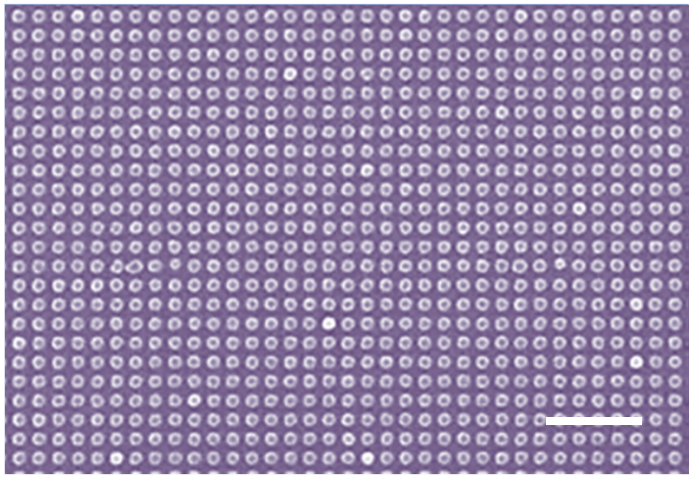

C

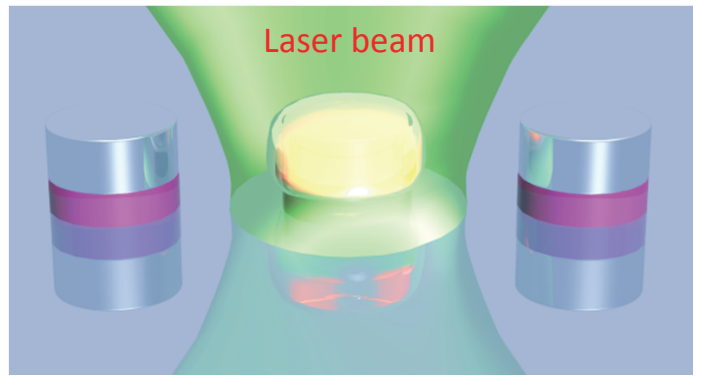

Figure 1: Plasmonic structures for colour printing. a, Schematic illustrations of the plasmonic metasurface and a unit cell with parameter settings, where the periodicity is $P=200 \mathrm{~nm}$, the thickness of $\mathrm{Al}$ is $T=20 \mathrm{~nm}$, the height of the pillars is $H=30 \mathrm{~nm}$ and the diameter of the disks is $D$. b, A top-view SEM image of a plasmonic metasurface. Scale bar: $1 \mu \mathrm{m}$. c, A schematic illustration of laser printing. The printing is governed by photo-thermal reshaping of the metasurface. 

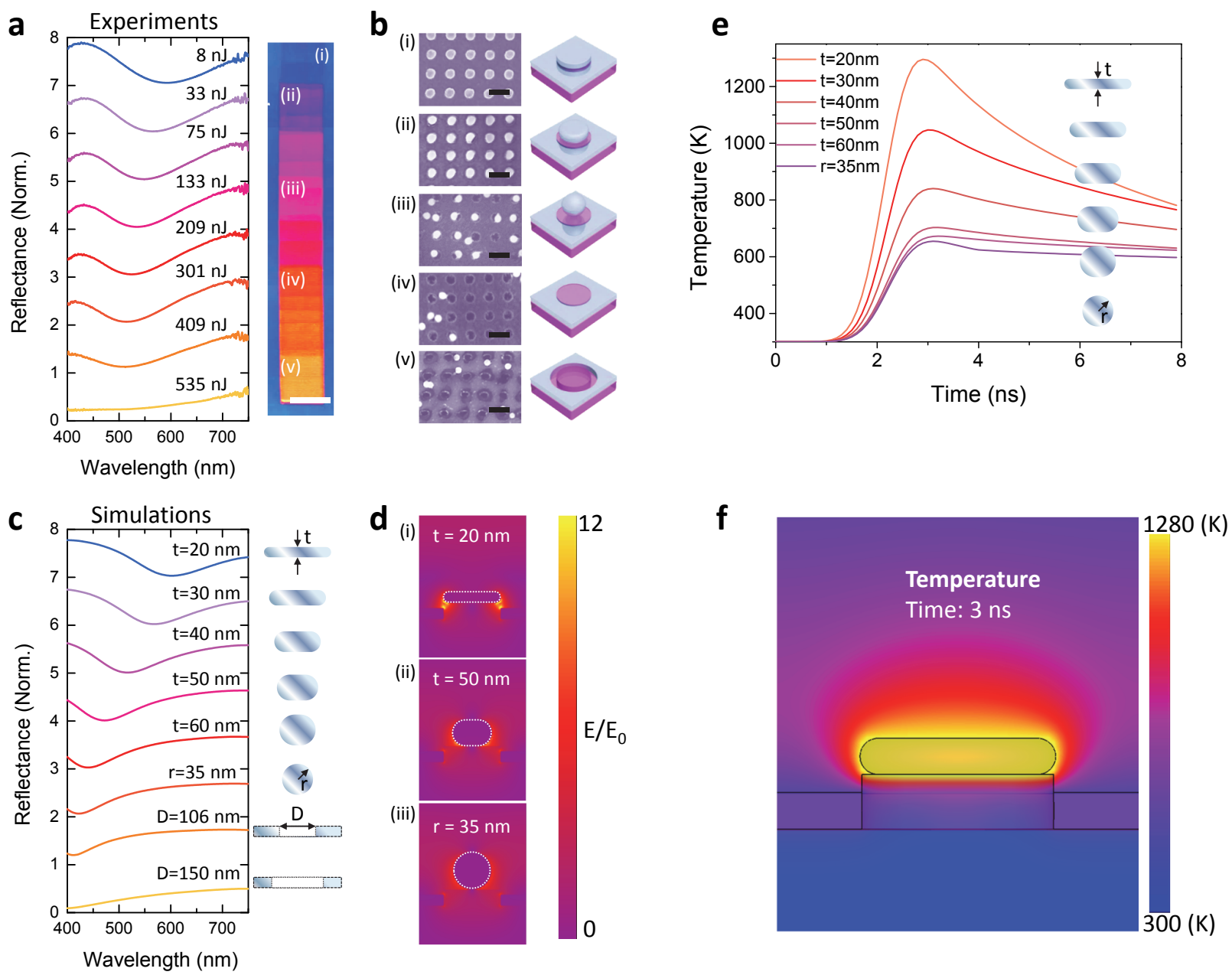

Figure 2: Spectral and geometry analysis and numerical simulations. a, Left, experimental spectra of the metasurface with a selected disk diameter, $D=106 \mathrm{~nm}$. The dips appear from SPRs under increasing laser dosage from 0 to $535 \mathrm{~nJ}$ of the single-pulse laser exposure ( $1 \mathrm{~ns}, \lambda=532 \mathrm{~nm}$, spot size: $50 \mu \mathrm{m})$. Right, corresponding colour squares of $500 \mu \mathrm{m} \times 500 \mu \mathrm{m}$ printed with a $10 \mu \mathrm{m}$ step size. Scale bar: $500 \mu \mathrm{m}$. b, Left, corresponding SEM images of colour areas in a, Labels (i)-(v). Scale bars: $200 \mathrm{~nm}$. The related pulse energies are 0 (unexposed), 33, 133, 301 and 535 nJ. Right, 3D artworks for demonstrating the corresponding morphology evolutions. c, Simulated spectra of models by imitating the morphology transformation in measurements. The cross-section demos in the right panel indicate the parameter settings. For clarity, the normalized spectra in (a) and (c) have been displaced by +1 . d, Simulated electric field enhancements (at $\lambda=532 \mathrm{~nm}$ ) for selected morphologies of (i) $t=20 \mathrm{~nm}$, (ii) $t=50 \mathrm{~nm}$ and (iii) $r=35 \mathrm{~nm}$ (sphere). e, Simulated temperature evolutions during the first initial nanoseconds for different structural morphologies, which gradually change the morphology from a disk shape into a spherical particle. The crosssectional views indicate the morphology changeland highlight the various geometrical parameters. f, The corresponding temperature distribution in the initial structure after $3.0 \mathrm{~ns}$, in the case where the initial thickness of the disk is $t=20 \mathrm{~nm}$. The incident pulse power and absorbed energy and the heat power density distribution are shown in Supplementary Figure S6. The 3D evolution of 
a
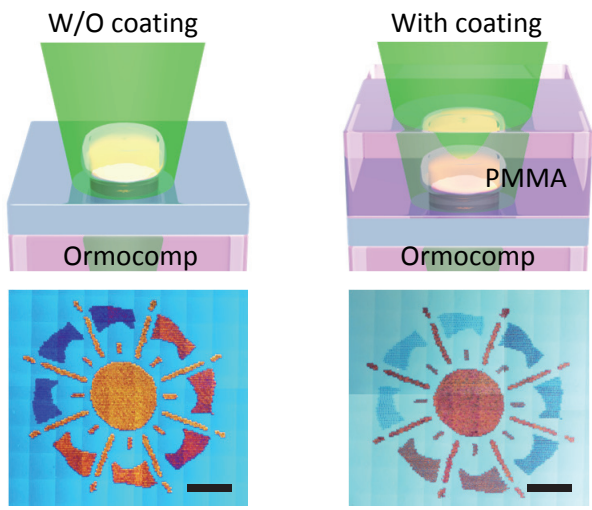

b

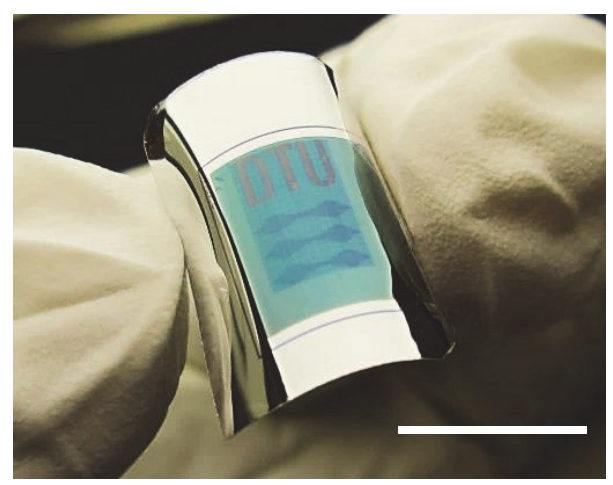

C
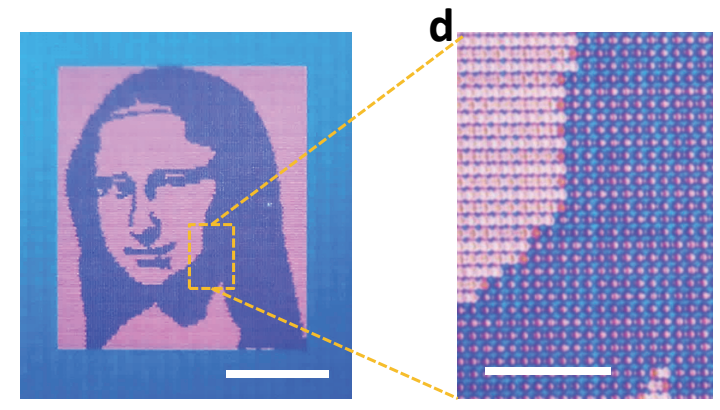

e
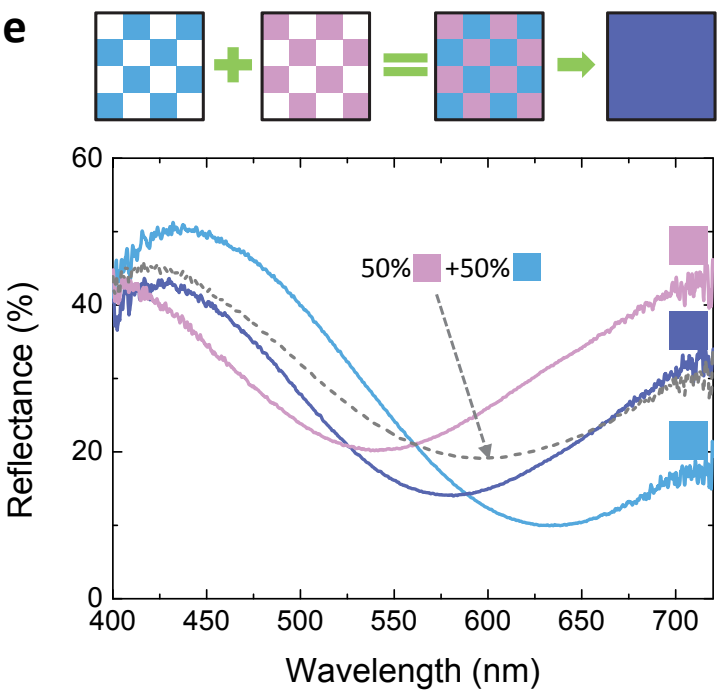

Figure 3: Flexible and robust samples for colour printing and colour mixing. a, Illustrations and examples of laser printing on samples without (left) and with (right) polymer coating. Scale bars: $500 \mu \mathrm{m}$. b, A large-scale sample with plasmonic colours printed by laser transmitted through the protecting polymer. Scale bar: $1 \mathrm{~cm}$. c, A printed portrait of "Mona Lisa" photographed by a camera with a macro-lens. Scale bar: $2 \mathrm{~mm}$. d, A magnified image taken by a microscope exhibits colour dots with different dimensions. Scale bar: $500 \mu \mathrm{m}$. e, Corresponding reflection spectra of selected pixels with different colours: the background cyan colour, the printed magenta colour and the mixed blue-violet colour. Mixed colour dots make a new colour and a new spectrum. 

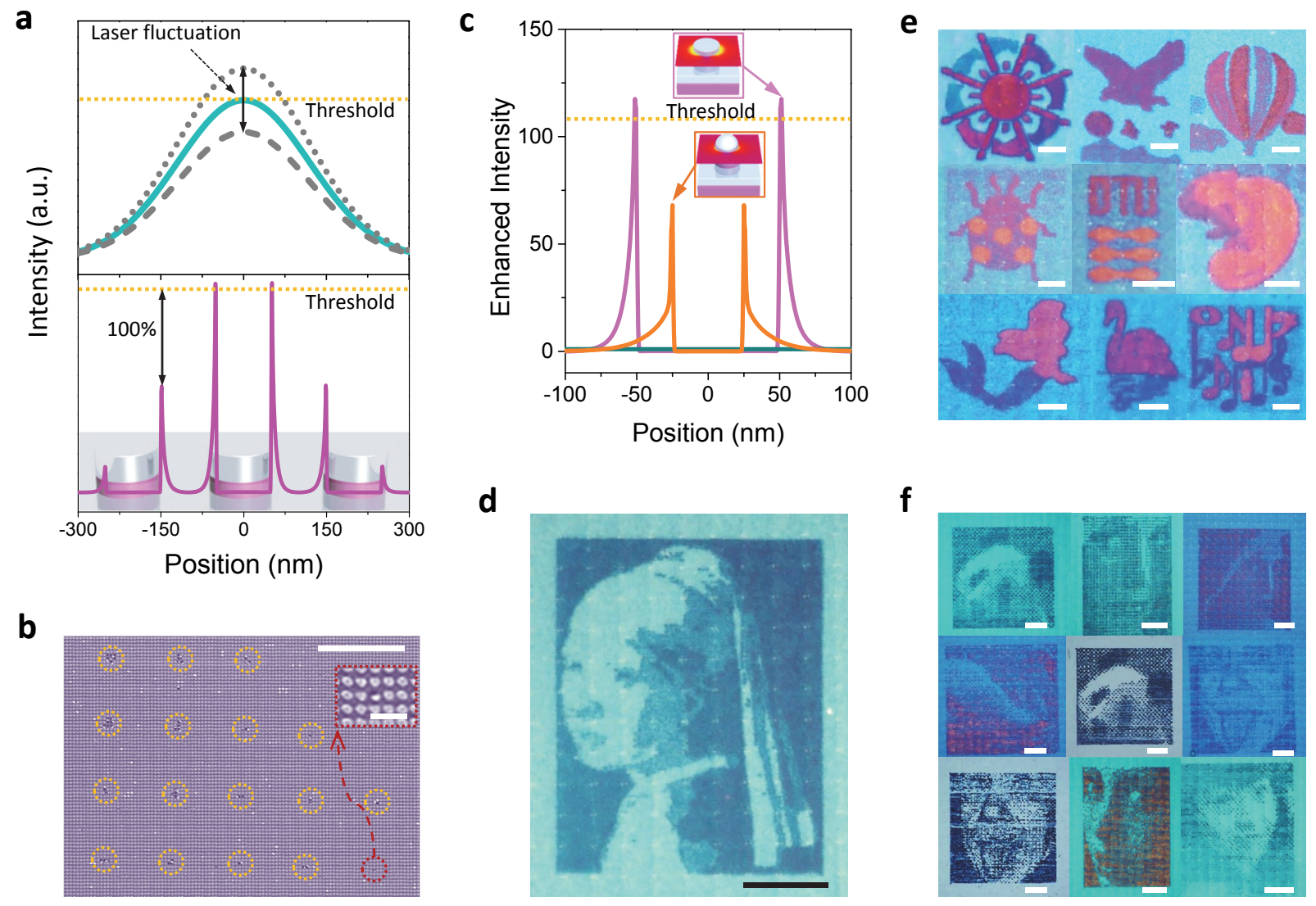

Figure 4: Laser printing with sub-diffraction-limit resolution. a, Upper, sub-diffraction-limit laser printing without plasmonic reconfiguration, where dashed, solid and dotted lines respectively denote the laser-pulse energy below, at and above the melting threshold (the yellow dashed line). Bottom, advanced sub-diffraction-limit printing where photon energy is redistributed with optical field confinement and enhancement by the plasmonic metasurface. $\mathbf{b}$, SEM image of voxels formed at exposure with different laser-pulse energies, which are gradually decreased from the upper left corner to the bottom right one. Scale bar: $5 \mu \mathrm{m}$. The inset reveals an interesting area in the red dashed circle (scale bar: $500 \mathrm{~nm}$ ). c, The field enhancements in the central section of the unit cells with a disk and a sphere, respectively. The insets show the corresponding 2D field distributions. d, A colour design in blue tone printed with single-unit-cell resolution (127,000 DPI). Scale bar: $10 \mu \mathrm{m}$. e, Printed images in different colour schemes with single-unit-cell resolution. The laser energy used for patterning were moderated to minimize influence for different printing channels, while strong power will incite crosstalks of plasmonic energy distribution between the neighboring unit cells and degrade the resolution. Scale bars: $5 \mu \mathrm{m}$. f, A collection of images printed by grayscale half-toning. The printing resolution is 63,500 DPI. Scale bars: $10 \mu \mathrm{m}$. 


\section{References}

1. Ebbesen, T. W., Lezec, H. J., Ghaemi, H. F., Thio, T. \& Wolff, P. A. Extraordinary optical transmission through sub-wavelength hole arrays. Nature 391, 667-669 (1998).

2. Maier, S. A. Plasmonics: fundamentals and applications (Springer, New York, NY 10013, USA, 2007).

3. Pendry, J. B., Schurig, D. \& Smith, D. R. Controlling electromagnetic fields. Science 312, 1780 (2006).

4. Leonhardt, U. Optical conformal mapping. Science 312, 1777 (2006).

5. Dean, N. Colouring at the nanoscale. Nat. Nanotechnol. 10, 15-16 (2015).

6. Kumar, K. et al. Printing colour at the optical diffraction limit. Nat. Nanotechnol. 7, 557-561 (2012).

7. Wu, Y.-K., Hollowell, A. E., Zhang, C. \& Guo, L. J. Angle-insensitive structural colours based on metallic nanocavities and coloured pixels beyond the diffraction limit. Sci. Rep. 3, 1194 (2013).

8. Roberts, A. S., Pors, A., Albrektsen, O. \& Bozhevolnyi, S. I. Subwavelength plasmonic color printing protected for ambient use. Nano Lett. 14, 783-787 (2014).

9. Tan, S. J. et al. Plasmonic color palettes for photorealistic printing with aluminum nanostructures. Nano Lett. 14, 4023-4029 (2014). 
10. Goh, X. M. et al. Three-dimensional plasmonic stereoscopic prints in full colour. Nat. Commun. 5, 5361 (2014).

11. Ellenbogen, T., Seo, K. \& Crozier, K. B. Chromatic plasmonic polarizers for active visible color filtering and polarimetry. Nano Lett. 12, 1026-1031 (2012).

12. Zeng, B., Gao, Y. \& Bartoli, F. J. Ultrathin nanostructured metals for highly transmissive plasmonic subtractive color filters. Sci. Rep. 3, 2840 (2013).

13. Cheng, F., Gao, J., Luk, T. S. \& Yang, X. Structural color printing based on plasmonic metasurfaces of perfect light absorption. Sci. Rep. 5, 11045 (2015).

14. Yokogawa, S., Burgos, S. P. \& Atwater, H. A. Plasmonic color filters for cmos image sensor applications. Nano Lett. 12, 4349-4354 (2012).

15. Chichkov, B. N., Momma, C., Nolte, S., von Alvensleben, F. \& Tünnermann, A. Femtosecond, picosecond and nanosecond laser ablation of solids. Appl. Phys. A 63, 109-115 (1996).

16. Knight, M. W. et al. Aluminum plasmonic nanoantennas. Nano Lett. 12, 6000-6004 (2012).

17. Olson, J. et al. Vivid, full-color aluminum plasmonic pixels. Proc. Natl. Acad. Sci. U.S.A. 111, 14348-14353 (2014).

18. Prodan, E., Radloff, C., Halas, N. \& Nordlander, P. A hybridization model for the plasmon response of complex nanostructures. Science 302, 419-422 (2003).

19. Clausen, J. S. et al. Plasmonic metasurfaces for coloration of plastic consumer products. Nano Lett. 14, 4499-4504 (2014). 
20. Wang, Y. M. et al. High aspect ratio 10-nm-scale nanoaperture arrays with template-guided metal dewetting. Sci. Rep. 5, 9654 (2015).

21. Chen, X., Chen, Y., Yan, M. \& Qiu, M. Nanosecond photothermal effects in plasmonic nanostructures. ACS Nano 6, 2550-2557 (2012).

22. Zywietz, U., Evlyukhin, A. B., Reinhardt, C. \& Chichkov, B. N. Laser printing of silicon nanoparticles with resonant optical electric and magnetic responses. Nat. Commun. 5, 3402 (2014).

23. Baffou, G., Quidant, R. \& García de Abajo, F. J. Nanoscale control of optical heating in complex plasmonic systems. ACS Nano 4, 709-716 (2010).

24. Zijlstra, P., Chon, J. W. M. \& Gu, M. Five-dimensional optical recording mediated by surface plasmons in gold nanorods. Nature 459, 410-413 (2009).

25. Kim, H. et al. Structural colour printing using a magnetically tunable and lithographically fixable photonic crystal. Nat. Photonics 3, 534-540 (2009).

26. Ulichney, R. Digital Halftoning (MIT Press, Cambridge, Massachusetts, USA, 1987).

27. Kawata, S., Sun, H., Tanaka, T. \& Takada, K. Finer features for functional microdevices. Nature 412, 697-698 (2001).

28. Barnes, W. L., Dereux, A. \& Ebbesen, T. W. Surface plasmon subwavelength optics. Nature 424, 824-830 (2003). 
29. Fang, N., Lee, H., Sun, C. \& Zhang, X. Sub-diffraction-limited optical imaging with a silver superlens. Science 308, 534-537 (2005).

30. Højlund-Nielsen, E., Greibe, T., Mortensen, N. A. \& Kristensen, A. Single-spot e-beam lithography for defining large arrays of nano-holes. Microelectron. Eng. 121, 104-107 (2014).

Acknowledgment This work was supported by the European Commission via the FP7MMP Integrated project PLAST4FUTURE (NMP2-SE-2012-314345). The authors thank C. Smith and K. T. Sørensen for technical support and W. Yan, J. Clausen and S. Xiao for fruitful discussions.

Author contributions X.Z., N.A.M. and A.K. conceived the ideas. X.Z. and E.H. fabricated the nanoimprinted samples. X.Z. performed the simulations. C.V. suggested and built the optical setup for laser printing. X.Z. and C.V. developed the codes. X.Z. implemented the laser printing and prepared the figures. A.K. and N.A.M. provided feedback on the experiments. All authors contributed to the writing of the manuscript.

Additional information Supplementary information accompanies this paper at www.nature.com/naturenanotechnology. Reprints and permission information is available online at http://npg.nature.com/reprintsandpermissions/.

Correspondence A.K. (Anders.Kristensen@nanotech.dtu.dk) or X.Z. (xizhu@ nanotech.dtu.dk) or N.A.M. (asger@mailaps.org).

Competing financial interests The authors declare no competing financial interests. 